\title{
Virtual prototyping of the solar tracking systems
}

\author{
C. Alexandru and M. Comşiț \\ Department of Product Design and Robotics \\ University Transilvania of Braşov \\ 29 Bd. Eroilor, 500036 Brasov, Romania \\ phone:+40 268 412921, fax:+40 268 472496, e-mail: calex@unitbv.ro, comsit@unitbv.ro
}

\begin{abstract}
The subject of the paper is now-a-days a priority on international level, because it provides alternatives to major problems resulting from the use of fossil resources: the limitative character and the pollution. The paper presents researches in the field of increasing the efficiency of the solar energy conversion by using tracking systems whose aim is to change the position of the solar panel correlated to the sun position for maximizing the radiation degree of use. Two main aspects are taken into consideration: optimizing the interaction between the mechatronic system components by integrating the control system in the mechanical model, beginning with the virtual prototype level, and reducing the cost \& time for the design process by replacing the traditional tests on physical models with the testing in virtual environment, using complex digital (software) models.
\end{abstract}

\section{Key words}

Solar panel, tracking mechanism, control system, virtual prototype.

\section{Introduction}

There is a fact that the fossil fuels (ex., gas, oil, coal) are limited and hand strong pollutants. In the last 15 years, the price of petroleum had tripled and the previsions on the medium term there are not quite encouraging. The increase of the emissions of carbon dioxide, responsible for the global warming and for the greenhouse effect, may have devastating results over the time on the environment.

The solution to the previously highlighted problems is the renewable energy, including the energy efficiency, the energy saving and systems based on clean renewable energy sources, like sun, wind and water. The concept of sustainable development have been enounced for the first time in 1987, in the Brundland Commission Report, and subsequent adopted at the political level, so in the Conference for Development and Environment from Rio de Janeiro (1992), the participant countries have undertaken to develop national strategies for sustainable development (The Program Agenda 21). The instruments for the implementation of the concept are in continuous development and represents major directions for research in the European projects FP6 and FP7.
The solar energy conversion is one of the most addressed topics in the fields of renewable energy systems. The sun is a giant nuclear fusion reactor and the energy it supplies is equivalent of about 27,000 times the total amount of energy presently produced from all other sources. As a result of the atmospheric phenomena involving reflection, scattering, and absorption of radiation, the quantity of solar energy that ultimately reaches the earth's surface is much reduced in intensity as it traverses the atmosphere. Finally, the total solar radiation received at ground level includes direct solar radiation and diffuse radiation [15].

\section{Tracking Systems}

The efficiency of the solar system depends on the degree of use and conversion of the solar radiation. When performing the energy balance on the solar panel, reference is done to the surface that absorbs the incoming radiation and to the balance between energy inflow and energy outflow. The rate of useful energy leaving the absorber is given by the difference between the rate of optical (short wavelength) radiation incident on absorber and the rate of energy loss from the absorber [8].

In these terms, there are two ways for maximizing the rate of useful energy: optimizing the conversion to the absorber level, and decreasing the losses by properly choosing the absorber materials; increasing the incident radiation rate by using mechanical tracking systems (the maximum degree of collecting is obtained when the incident radiation is perpendicular on the active surface). Basically, the tracking systems are mechanical devices (i.e. mechanisms), driven by motors or actuators, which orient the panel in order to follow the sun path on the sky. The orientation of the solar panels may increase the efficiency of the conversion system up to $50 \%[1,2]$.

The orientation principle is based on the input data referring to the position of the sun on the sky dome (fig. 1). For the highest conversion efficiency, the sunrays have to fall normal on the receiver so the system must periodically modify its position in order to maintain this relation between the sunrays and the panel. The positions of the Sun on its path along the year represent an input data in designing the solar trackers. 
The Earth describes along the year a rotational motion following an elliptical path around the sun. During one day ( 24 hours), the Earth also spins around its own axis describing a complete rotation, which generates the sunrises and the sunsets. The variation of the altitude of the sun on the celestial sphere during one year is determined by the precession motion, responsible for a declination of the Earth axis in consideration with the plane of the elliptic yearly path; the value of this angle is $23.5^{\circ}$ (see fig. 1); this motion generates the seasons because of the alternative exposure of the northern and southern hemisphere to the sunrays trajectory.

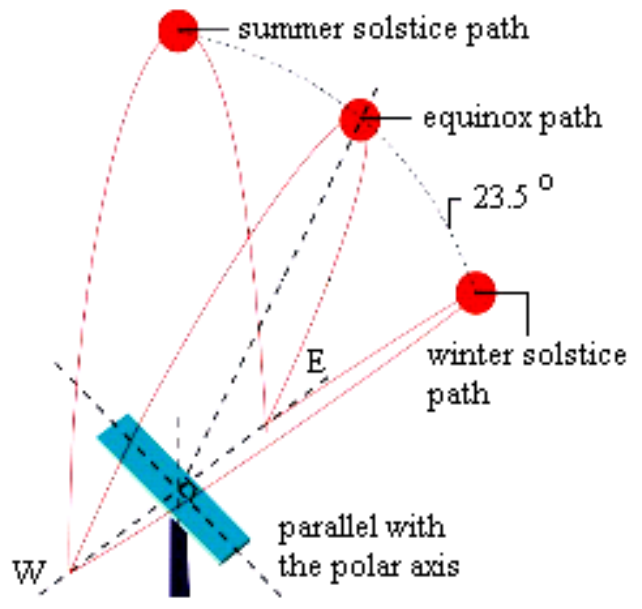

Fig. 1. Solar orientation principle.

Consequently, for the design process of the tracking systems, there are taken into consideration two rotational motions: the daily motion $\left(360^{\circ}\right)$, and the yearly precession motion $\left(2 \times 23.5^{\circ}\right)$. In these conditions, there are two fundamental ways to track the sun - by one axis, or by two axes. This fact determines two types of solar tracking systems (fig. 2): single axis (a), and dual-axis trackers (b).

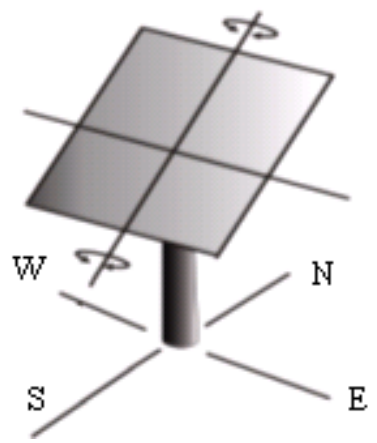

a.

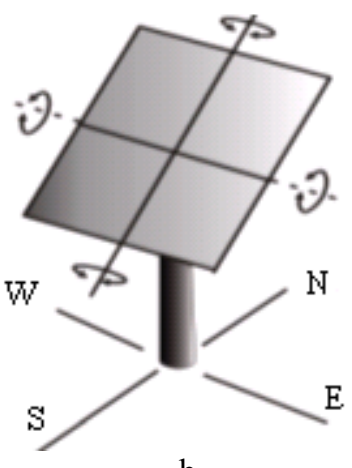

b.
Fig. 2. Basic solar trackers.

The single-axis tracking systems pivot on their axis to track the sun, facing east in the morning and west in the afternoon. The tilt angle of this axis equals the latitude angle of the loco because this axis has to be always parallel with the polar axis. In consequence for this type of tracking systems is necessary a seasonal tilt angle adjustment.
The two-axis trackers combine two rotational motions, so that they are able to follow very precisely the sun path along the period of one year. That's why dual axis tracking systems are more efficient than the single one, but also more expensive because they are using electrical and mechanical parts. In this case, there are two independent motions: the daily motion, and the seasonal motion, respectively.

\section{Analysis Algorithm}

The modern design process of the tracking systems involves conceptual and functional design, command \& control, digital mock-up, virtual prototyping and testing [14]. The conceptual design has as main objective to establish the best product concept (in the given conditions, by performing an efficient management of information picked by the science, technology, economy, market, culture, legislation, policy) [10]. The functional design involves identifying, modelling and evaluating the operational performances of the tracking systems, and the deviations from the imposed characteristics, with other words the mode in which the tracking systems responses to the design requirements.

The analysis flow-chart of the tracking mechanisms is shown in figure 3 . The kinematic model contains the rigid parts, which are connected through geometric constraints, and the specific geometric parameters; the input is made using kinematic restrictions (motion generators) that controls the angular or linear position / velocity of the driving elements. The inverse dynamic model includes the kinematic model and, in addition, the external and internal loading; this model is used to determine the turning moment or force applied by the driving element. Finally, the dynamic model includes the inverse dynamic model, but the input is made through the above-determined torque / force; the aim is to evaluate the "real" behaviour of the tracking system.

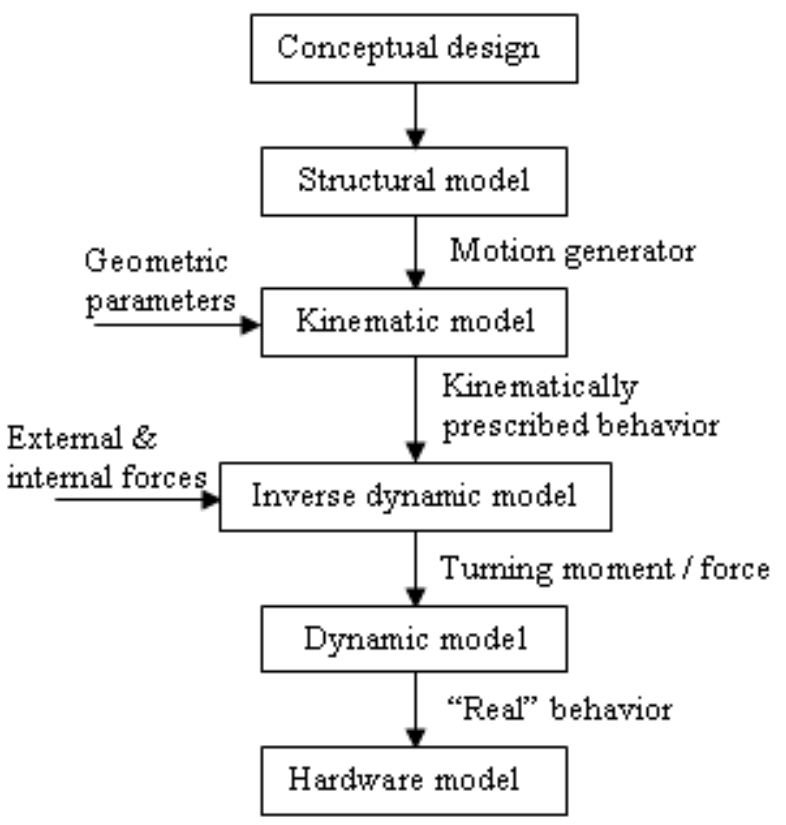

Fig. 3. Analysis flow-chart. 
Determining the real behaviour of the tracking systems is a priority in the design stage since the emergence of the computer graphic simulation. Important publications reveal a growing interest on analysis methods for multibody systems (MBS) that may facilitate the selfformulating algorithms, having as main goal the reducing of the processing time in order to make possible real-time simulation $[5,12,13,16]$. In the last decade, a new type of studies was defined through the utilization of the MBS software: Virtual Prototyping. This technology consists mainly in conceiving a detailed model and using it in a virtual experiment, in a similar way with the real case. No longer is it necessary to wait months to build a hardware prototype, instrument it, run tests on it, and make a small number of expensive modifications to it in order to assess proposed design changes [6].

The traditional CAD/CAM/CAE practices focused on a concept referred to as art-to-part [11]. Nearly all software activities were oriented toward the design, development, and manufacturing of higher quality parts. Unfortunately, optimal part design rarely leads to optimal system design. The interaction of form, fit, function, and assembly of all parts in a product is a major contributor to overall product quality. The big opportunity to increase quality and reduce time and cost has now shifted to the system level. Specifically, the system-level solutions include digital mock-up tools to investigate product form and fit, and functional virtual prototyping tools to assess product function and operating performance.

Basically, the virtual prototyping platform includes CAD, MBS and FEA programs. The CAD environment is used to create the geometric model of the system, which contains information about the mass \& inertia properties of the rigid parts. The MBS software, which represents the central component of the virtual prototyping platform, is used for analyzing, optimizing and simulating the kinematic and dynamic behaviour of the mechanism. The FEA software is used for modelling flexible components, which allows capturing inertial and compliance effects during simulation, and predict loads with greater accuracy, therefore achieving more realistic results.

In addition, a modern virtual prototyping platform includes specific software for product data management (PDM). This technique is the glue that enables the main components of the virtual prototyping platform to be successful by making all of the up-to-date component data readily available and manageable [11].

The active tracking systems are mechatronic systems, which integrates mechanics, electronics and information technology. The mechatronic systems are built-up with some units with basic functions, which are made to interact between them in order to form a complex system with a given functionality. The component that increases the performance of the mechatronic system is the control software, which is integrated in the electronic part [9].

In the typical design process of a mechanical system with controls, the mechanical designer and the controls designer work from the same concept, but use different sets of software tools. The result is that each designer produces a model for the same problem. Each design is then subject to verification and testing, and the first time the two designs are brought together is during physical prototype testing. If a problem occurs during the interaction between the controls design and the mechanical design, the engineers must refine the control design and/or the mechanical design, and then go through the entire verification process.

Integrating the control system in the mechanical model, the two designers can share the same mechanical model (fig. 4); they can also verify from one database the combined effects of a control system on a nonlinear, nonrigid model. The physical testing process is greatly simplified, and the risk of the control law being poorly matched to the real system is eliminated. This approach is called concurrent engineering [4].

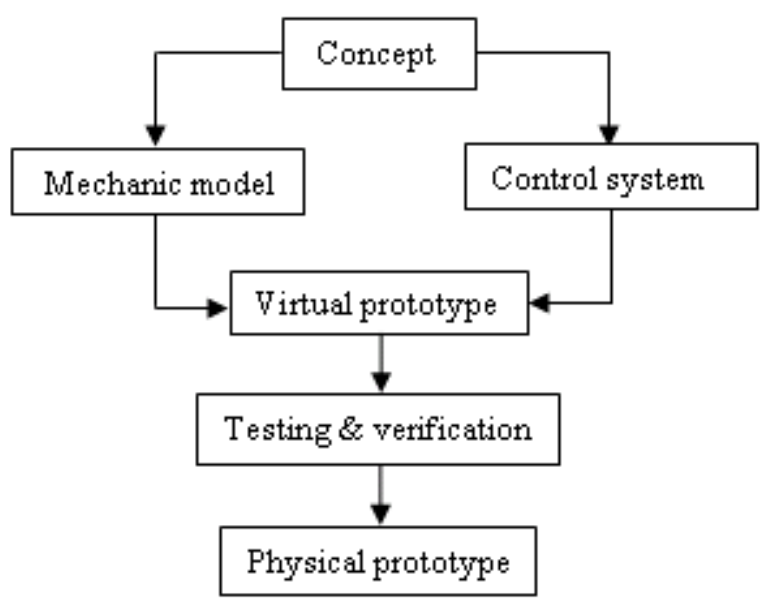

Fig. 4. Concurrent engineering model.

In these conditions, the virtual prototyping platform has to include a control software product, which directly exchanges information (import - export) with the MBS software; the output from the MBS model is input for the control system and vice-versa. In the concurrent engineering philosophy, the simulation algorithm of the tracking system, involves the following steps [7]:

- regarding the MBS environment: designing the mechanical model (including bodies, joints, elastic and damping elements, external forces and torques); analyzing the dynamic model; identifying the inputs and outputs, which complete a closed loop between the MBS model and the control application; exporting the obtained model;

- regarding the control application: importing the mechanical model block; explaining the mechatronic system trajectory in the task space and defining the input block diagram (the synthesis of the reference signals); designing the control system block diagram; designing the controller and the interface electric circuits; simulating the mechatronic system.

This simulation process creates a closed loop in which the control inputs from the control application affect the MBS simulation, and the MBS outputs affect the control input levels [17]. 
In these terms, the virtual prototyping platform that was used in our paper in shown in figure 5. The platform integrates licensed software, as follows: CAD - CATIA, MBS - ADAMS, FEA - NASTRAN/PATRAN, Control MATLAB, PDM - SMARTEAM.

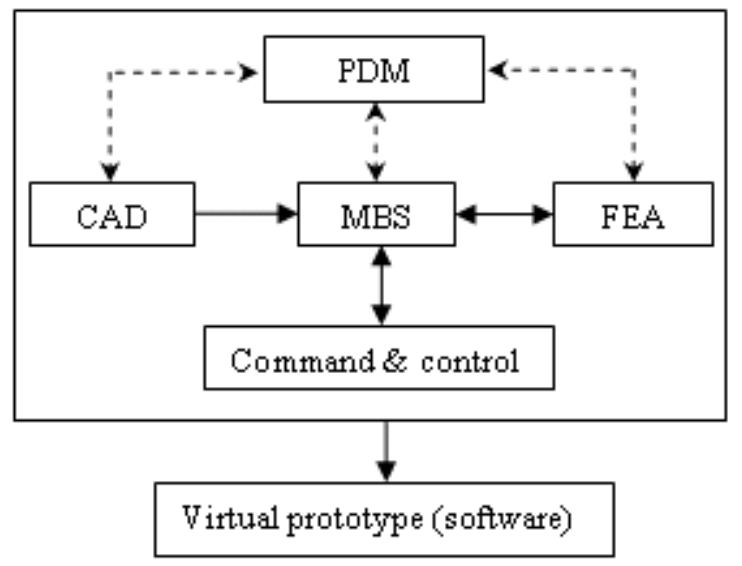

Fig. 5. Virtual prototyping platform

\section{Case Study}

In order to apply the above-described design algorithm, a dual-axis tracking system, with two degrees of freedom, has been taken into consideration. The tracking system is used for following the sun in both polar and seasonal directions, keeping the sun's rays normal to the platform surface. The daily motion is directly driven by a rotary motor that is able to develop an angular displacement up to 180 degrees. The seasonal motion is driven by a hydraulic actuator that changes the panel elevation.

The 3D-solid model was made using CAD software, namely CATIA. The geometry was transferred to ADAMS/View using the STEP file format, through ADAMS/Exchange module. The virtual model of the tracking system, shown in figure 6 , is designed so that it has five parts, which are connected one with other, respectively to ground, using the following geometric constraints: base (1) fixed on the ground; intermediary element (2) mounted on the base - revolute joint; hydraulic cylinder (4) to base - revolute joint; hydraulic piston (5) to intermediary element - revolute joint; cylinder to piston - translational joint; motor fixed on the intermediary element; panel (3) to motor (and on the intermediary element) - revolute joint.

For the kinematic model of the tracking system, there are two motion generators, which are applied in the revolute joint between intermediary element and the solar panel, and in the translational joint between the hydraulic piston and cylinder. For the dynamic model, the motion generators are replaced with external torque and axial force that drives the panel, which are computed by control systems, based on the error between the actual panel position and the desired position. This is more realistic than attaching motion generators and driving the motion directly; at the same time, we can look at issues related to motor size in an actual tracking system.
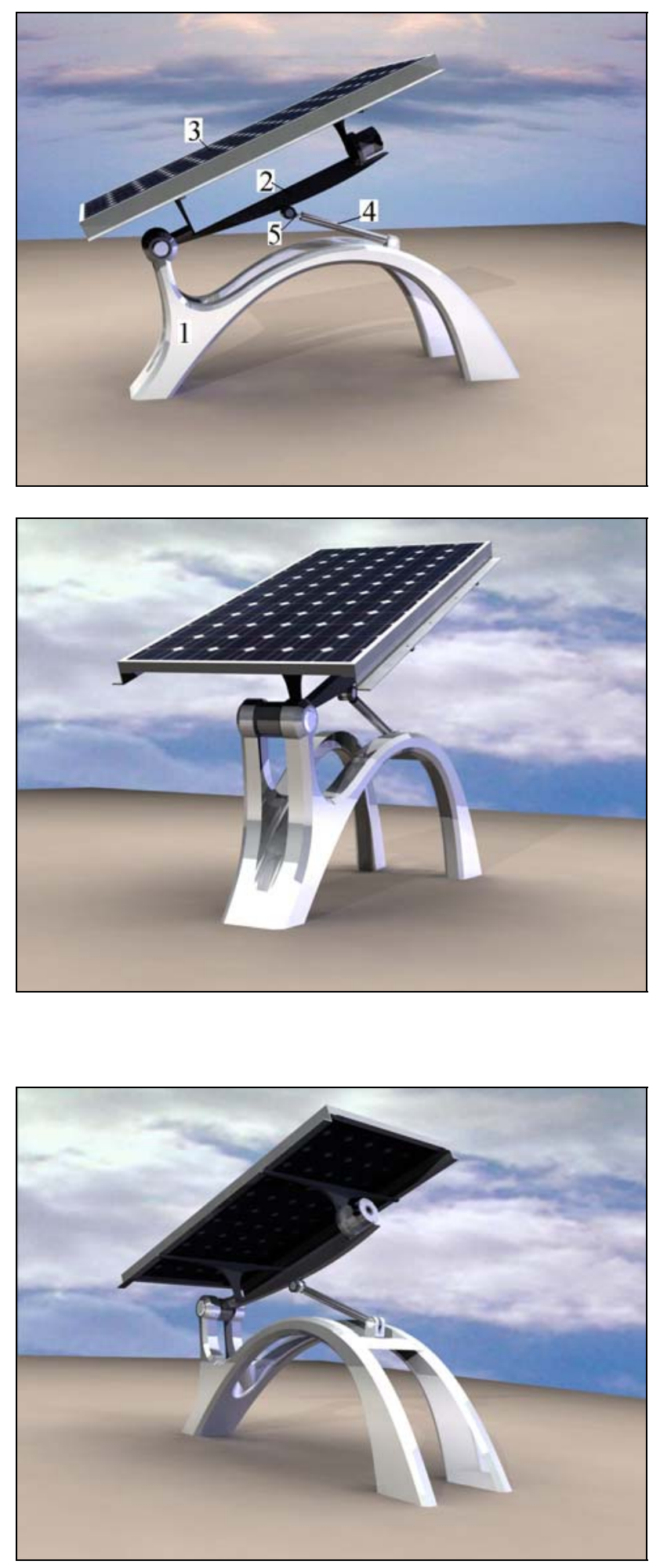

Fig. 6. Virtual prototype of the solar tracker.

For this paper, only the daily motion is considered, so that we are looking for the polar control torque that is applied to the platform. The control system is developed using ADAMS/Controls and MATLAB/Simulink. ADAMS/Controls is a plug-in to ADAMS/View that allows integrating motion simulation and controlling system design in the virtual model. ADAMS/Controls allow connecting the ADAMS mechanical model to block diagrams that are developed with control applications [17]. 
For driving the solar panel, a DC motor is used, so that the objective is to control the angular velocity of the rotor, which is perturbed with the motor torque. The angular velocity of the rotor determines in fact the daily position (polar angle) of the panel. The motor torque is obtained having in view the mechanical model of the tracking system, on which acts the external environment. The four-step process of combining controls with a mechanical system involves [3]: build the mechanical model, which includes all necessary geometry, constraints, forces; identify the ADAMS inputs and outputs - the outputs describe the variables that go to the controls application, and the inputs describe the variables that come back into ADAMS; build the control system block diagram, in MATLAB/Simulink, and include the ADAMS plant in the block diagram; simulate the combined mechanical model and control system.

In our case, the input to the solar tracking system model is the angular velocity of the rotor. The output, which will be transmitted to the controller, is the control torque. ADAMS/Controls and MATLAB communicate by passing state variables back and forth (fig. 7). Therefore, it is necessary to define the input and output variables of the model, and the functions that those inputs and outputs reference, with a set of ADAMS state variables.



Fig. 7. Combining ADAMS with MATLAB.

For the input state variable, representing the angular velocity of the rotor, the run-time function is 0.0 during each step of the simulation, because the velocity will get its value from the control application. The run-time function for the input variable is VARVAL(rotor velocity), where VARVAL is an ADAMS function that returns the value of the given variable [16]. In other words, the input angular velocity gets its value from the input variable. For the output state variable, representing the motor torque, the run-time function returns the sum of torques on body at location.

The next step is for exporting the ADAMS plant files for the control application. The Plant Input refers the input state variable (rotor_velocity), and the Plant Output refers the output state variable (control_torque). The input and output information are saved in a specific file for MATLAB (*.m). ADAMS also generates a command file (*.cmd) and a dataset file (*.adm) that will be used during the simulation. With these files, the control block was created in Simulink, in order to complete the link between the control and mechanical systems (fig. 8).

The control system is designed in order to rotate the solar panel with equal steps (15 degrees at every hour), around the polar axis, and allows actuating brakes. These steps may have variable period of driving that allow an energy consumption comparison between different values of actuating time. ADAMS accepts the rotor velocity from MATLAB and integrates the mechanical model in response to them. At the same time, ADAMS provides the control torque for MATLAB to integrate the Simulink model. This simulation process creates a closed loop in which the control input from MATLAB affect the ADAMS simulation, and the ADAMS outputs affect the control input level.

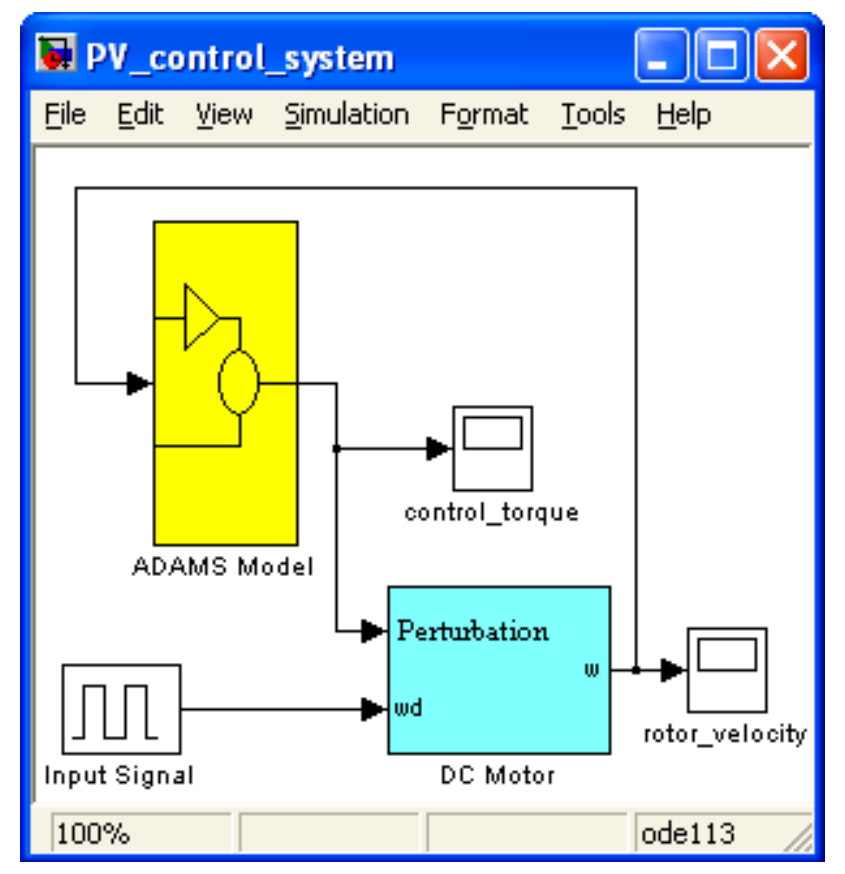

Fig. 8. Control system block.

Connecting the mechanical model with the control system, specific results are obtained in order to evaluate the dynamic behavior of the tracking system. For example, in figure 9, there are presented the time history variations of the control torque, rotor velocity, and panel polar position.

In addition, by integrating in the virtual prototyping platform a specific software for designing and simulating solar systems (ex. VALENTIN PV-SOL \& T-SOL), the functionality of the tracking system can be evaluated from energetic balance point of view (the contribution of energy that is obtained by tracking versus the material and energetic cost prices, including the energy consumed by the driving motors $\&$ actuators). This subject will be at length developed in a future paper.

\section{Conclusions}

The application presented in this paper is a typical example of virtual prototyping of the solar tracking systems by using multibody systems environment. One of the most important advantages of this kind of simulation is the possibility to perform virtual measurements in any point and/or area of the system and for any parameter 
(motion or force). This is not always possible in the real cases due to the lack of space for transducers placement or lack of appropriate transducers. This helps us to make quick decisions on any design changes without going through expensive prototype building and testing.


Fig. 9. Dynamic characteristics.

The result of virtual prototyping is that we are much better equipped to manage the risks inherent in the product development cycle. With virtual prototyping, behavioral performance predictions are obtained much earlier in the design cycle, thereby allowing more effective and cost efficient design changes and reducing overall risk substantially.
Concluding, the virtual prototyping brings several advantages: reduce the time and cost of new product development, reduce the product cycles, reduce the number of expansive physical prototypes and experiment with more design alternatives, increase quality and efficiency, and finally improve product.

\section{Acknowledgement}

The Romanian Ministry of Education financially supported the publishing of this paper in the frame of the CNCSIS research grant - code 418/2006.

\section{References}

[1] S. Abdallah and S. Nijmeh, "Design construction and operating of one-axis sun tracker", Journal of Applied Science, Vol. 4, pp. 45-53, 2002.

[2] S. Abdallah and S. Nijmeh, "Two-axis sun tracking with PLC control", Energy Conversion and Management, No. 45, pp. 31-39, 2004.

[3] C. Alexandru and C. Pozna, "Dynamics of mechanical systems using virtual prototyping tools", Transilvania University Publisher, Braşov (2003).

[4] R. Dorf and R. Bishop, "Modern control systems", Prentince Hall, London (2001).

[5] E.J. Haug, "Computer aided kinematics and dynamics of mechanical systems", Allyn and Bacon, New York (1989).

[6] E.J. Haug a.o., "Virtual prototyping simulation for design of mechanical systems", Transaction of ASME, No. 117, pp 63-70, 1995.

[7] V. Maties and D. Mândru, "Mechatronic technology and education", Todesco Publisher, Cluj-Napoca (2001).

[8] S. Odeh a.o., "Design of a single-axis tracking collector for moderate temperature applications", in Proceedings of the 14-th ISES Conference EUROSUN'2004, pp. 527-532.

[9] K. Ogata, "Discrete time control system", Prentince Hall, London (1995).

[10]G. Pahl and W. Beitz, "Engineering design", Springer-Verlag, Berlin (1996).

[11]R. Ryan, "Functional virtual prototyping", Mechanical Dynamics Publisher, Michigan (2001).

[12]W.O. Schiehlen, "Advanced multibody systems dynamics", Kluwer Academic, Dordrecht (1993).

[13]A. Shabana, "Dynamics of multibody systems", John Wiley \& Sons, New York (1988).

[14]D.G. Ullman, "The mechanical design process", McGrow-Hill, Columbus (1997).

[15]I. Vişa and M. Comşiț, "Tracking systems for solar energy conversion devices", in Proceedings of the 14-th ISES International Conference EUROSUN'2004, pp. 783-788.

[16]I. Vişa, "Mechanical systems modelling as multibody systems in product design", in Proceedings of PRASIC'02, pp. 255-263.

[17]*** "Getting started using Adams/View", MSC Software Publisher, Santa Ana (2005).

[18]*** "Getting started using Adams/Control", MSC Software Publisher, Santa Ana (2005). 